\title{
Front-end electronics for the ALICE dimuon trigger RPCs
}

\author{
Philippe Rosnet and Laurent Royer, for the ALICE Collaboration
}

\begin{abstract}
A dedicated front-end electronics has been developed for the trigger chambers of the ALICE muon spectrometer under construction at the future $\mathrm{LHC}$ at CERN. These trigger chambers are based on RPCs (Resistive Plate Chambers) working in streamer mode. The number of electronics channels (about 21000) and the fact that RPC pulsed signals have specific characteristics have led to the design of a 8 channels front-end ASIC using a new discrimination technique. The principle of the ASIC is described and the radiation hardness is discussed. Special emphasis is put on production characteristics of about 4000 chips.
\end{abstract}

Index Terms-LHC, ALICE, QGP, quarkonia, RPC, streamer, front-end, ASIC.

\section{INTRODUCTION TO PHYSICS CHALLENGE}

ALICE [1] (A Large Ion Collider Experiment) is a detector designed for the study of nucleus-nucleus collisions at the future LHC (Large Hadron Collider). Its physics program will address question concerning QCD (Quantum ChromoDynamics) of hot and dense nuclear matter produced in central heavy ion collisions at a center of mass energy of $5.5 \mathrm{TeV}$. The main goal of ALICE is to characterize a deconfined state of matter called the Quark-Gluon Plasma (QGP) [2]. One of its most promising probes is the production of quarkonia $(J / \Psi$ or $\Upsilon)$, which is expected to be decreased by color screening in the QGP [3].

The role of the ALICE forward spectrometer [4] is to reconstruct quarkonia in their dimuon channel in an angular acceptance of $\left[2^{0}, 9^{0}\right]$. To reach this physics goal, the spectrometer consists of a front and small angle (beam shielding) absorber, a set of high resolution tracking chambers, a dipole magnet, an iron wall (muon filter) and a trigger system.

\section{MUON TRIGGER SYSTEM}

The trigger system is composed by two stations of two detector planes of about $6 \times 6 \mathrm{~m}^{2}$ separated by one meter. The detectors are RPCs (Resistive Plate Chambers) [5] working in streamer mode at a high voltage value of about $8 \mathrm{kV}$ [6]. The necessary granularity implies to use strips of about 1, 2 and $4 \mathrm{~cm}$ width projective to the interaction point in each of the four planes, for a total of twelve different strip widths. Two perpendicular strip planes are used on each RPC (one on each side of the gas gap) to allow a three dimensional hits reconstruction. The total number of channels is then close to 21000.

Talk given by Ph. Rosnet, LPC Clermont-Ferrand, CNRS/IN2P3 and Université Blaise Pascal, 63177 Aubière Cedex, France (Email: rosnet@in2p3.fr).
The muon trigger is involved in the level 0 (L0) of the general ALICE trigger system [7]. The timing constraint required by the L0 is a muon trigger signal delivered each $25 \mathrm{~ns}(40 \mathrm{MHz})$ to the central trigger processor less than $800 \mathrm{~ns}$ after the collision. The architecture of the muon trigger electronics consists of the front-end electronics which picks-up and processes the RPC pulses and sends logical signals to the local trigger electronics (234 boards). The role of the local trigger is to store all signals (in a sequence of bit-patterns) in a pipeline memory and to identify single tracks with a transverse momentum above predefined cuts (by using a look-up-table) by means of a dedicated algorithm located in FPGAs (Field Programmable Gate Arrays). The regional (16 boards) and next the global ( 1 board) triggers collect the information from the local boards in order to select single or dimuon events from the full system.

\section{FRONT-END ELECTRONICS PRINCIPLE}

As shown in Fig. 11 the RPC pulses are composed of two peaks, called precursor and streamer. The precursor has the particularity to be synchronous with the particle crossing the RPC. The second peak is characterized by a variable time jitter with respect to the precursor, but with a large amplitude as expected for a streamer pulse.

\section{A. ASIC main characteristics}

To take advantage of these properties a new discrimination technique, called ADULT [8] (A DUaL Threshold), has been developed and implemented in a 8 channels ASIC using the AustriaMicroSystems BiCMOS $0.8 \mu \mathrm{m}$ technology [9]. This bipolar technology, with very low offset, is well adapted to trigger on small amplitude signals. The necessary number of ASIC for equipping all the muon trigger chambers is 2624 (not including spares).

The schematic of a single electronics channel is shown in Fig. 2 The precursor detection with a low threshold (typically $10 \mathrm{mV}$ ) provides a good time reference, and the streamer validation with a high threshold (about $100 \mathrm{mV}$ ) takes advantage of this RPC working mode: a large signal/noise ratio and a small cluster size (defined as the mean number of adjacent strips fired). A coincidence of these two discriminator output signals defines a hit of a strip. The time resolution obtained with this technique is comparable to the avalanche, which is about $1 \mathrm{~ns}$ as compared to 3 ns typically obtained with a single discriminator in streamer mode. The delayed (15 ns) low threshold comparator defines the reference time by using 


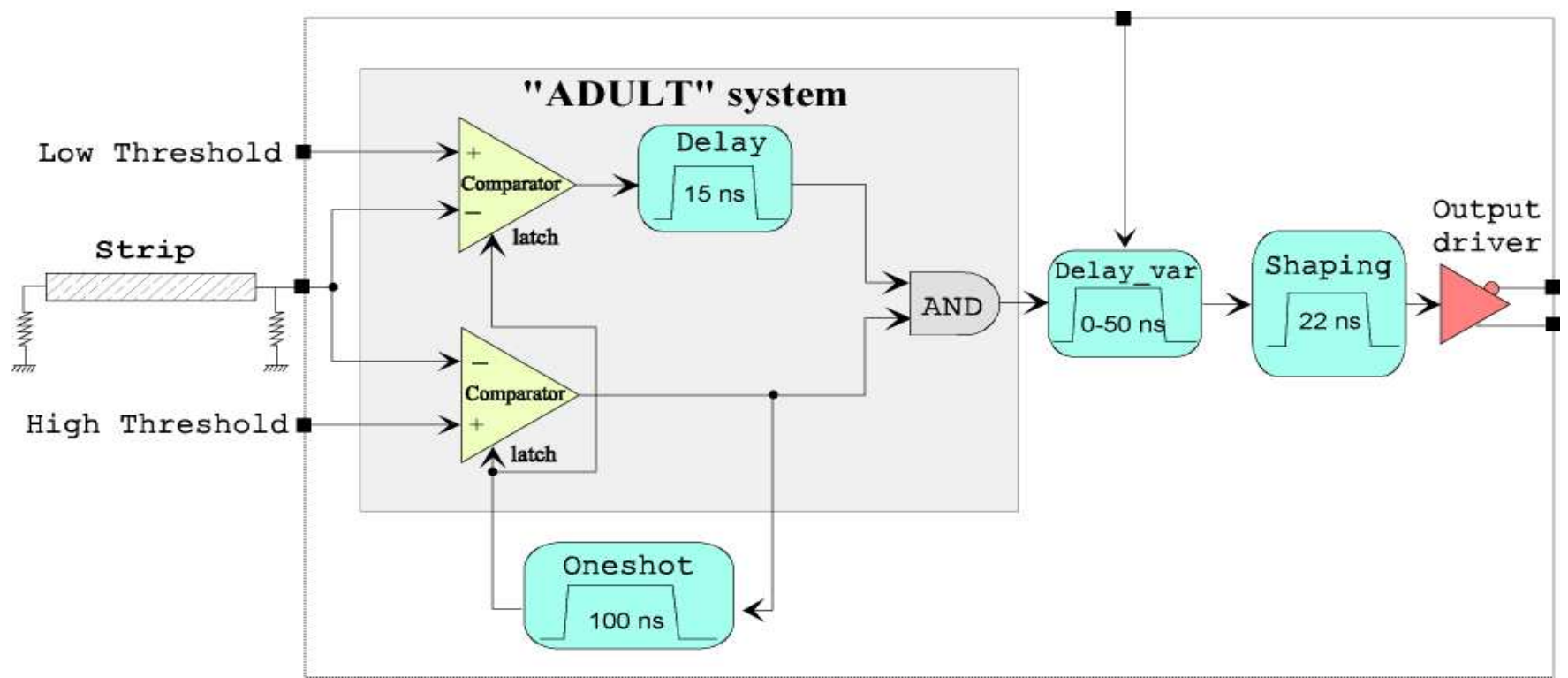

Fig. 2

BLOCK DIAGRAM OF A SINGLE ELECTRONICS CHANNEL.
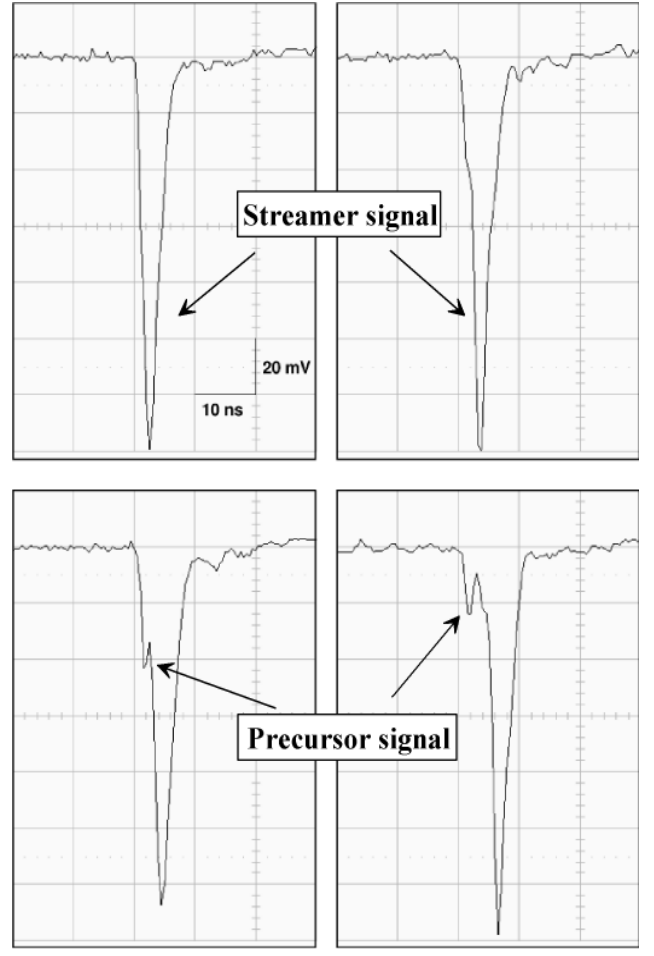

Fig. 1

TYPICAL PULSES PICKED-UP ON A SINGLE GAS GAP RPC OPERATING IN STREAMER MODE, WITH A DIGITAL SCOPE (1 GHZ BANDWIDTH) VIA A SHORT BNC CABLE (50 $\Omega$ IMPEDANCE) SYNCHRONIZED WITH PARTICLES CROSSING SCINTILLATOR PLATES READOUT BY PHOTO-MULTIPLIERS. the precursor as long as the streamer jitter is less than about $15 \mathrm{~ns}$. The oneshot function is used to latch the two comparators during $100 \mathrm{~ns}$, via a monostable, when a streamer signal has been validated to avoid re-triggering. A remote control delay, up to $50 \mathrm{~ns}$, common to the 8 channels of the ASIC is tuned by an external DC voltage and allows to adjust the timing of the ASIC. The signal is then converted into a (22-23) ns logical LVDS signal in order to drive the signal through a twisted pair cable from the RPCs to the local electronics trigger boards.

The main characteristics of an ADULT ASIC, as shown in Fig. 3, are the following:

- AustriaMicroSystems BiCMOS $0.8 \mu \mathrm{m}$ technology,

- 8 electronics channels,

- die surface equal to about $8 \mathrm{~mm}^{2}$,

- plastic packaging type PLCC 52 pins,

- power consumption: $10 \mathrm{~mW} /$ channel for $-2 \mathrm{~V}$ and $80 \mathrm{~mW} /$ channel for $+3.5 \mathrm{~V}$.

\section{B. Front-end boards supporting the ASIC}

The ASIC is implemented on a dedicated board developed to pick-up the signals provided by the RPC strips, as shown in Fig. 4 Twelve different strip widths are necessary to equip the full detector, but only six boards with different mechanical characteristics are needed. Two ASICs are used on the boards corresponding to strips of $1 \mathrm{~cm}$ width (16 channels) while the boards associated with strips of width $2 \mathrm{~cm}$ or $4 \mathrm{~cm}$ ( 8 channels) contain only one ASIC. Each single gas gap RPC provides positive pulses on one side and negative pulses on the other side of the gas gap. Then, the two polarities are implemented 

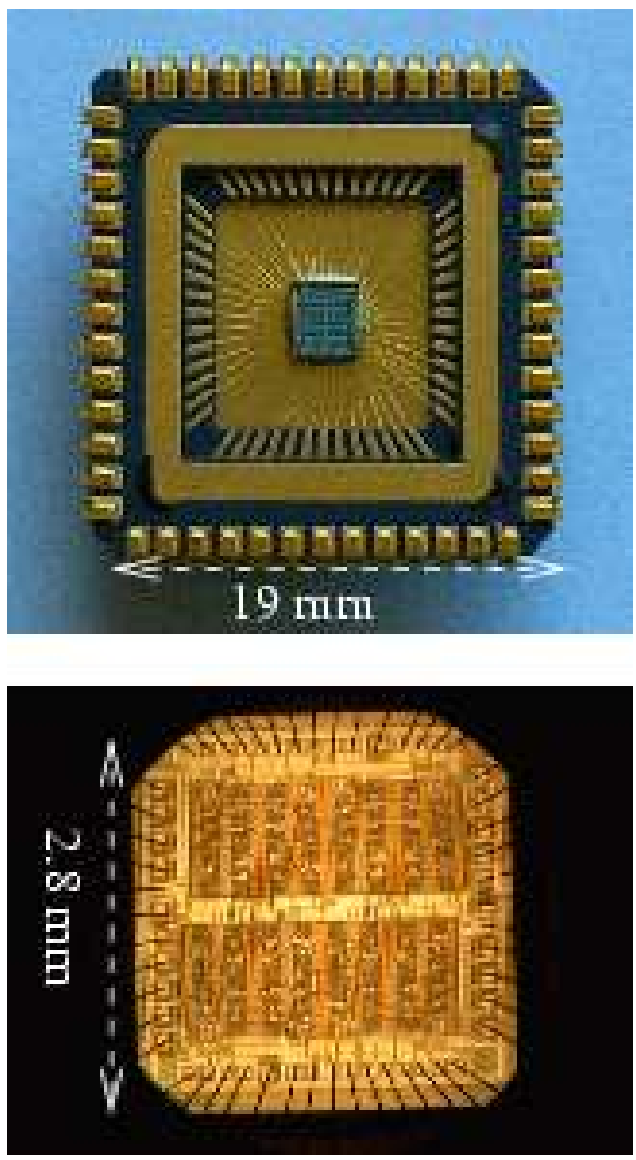

Fig. 3

ASIC AFTER PACKAGING (UPPER PICTURE) AND VIEW OF THE SILICON PART WITH A MICROSCOPE (LOWER PICTURE).

at the cabling level on the front-end boards. Furthermore, the different distances between the boards and the trigger electronics located in racks above the chambers (6 meters high) lead to different output cable length. These differences were compensated for on the boards by implementing delays in step of $7.5 \mathrm{~ns}$ corresponding to $1.5 \mathrm{~m}$ of cable. To summarize, the total number of different boards after cabling is equal to ten and each board can be configured with five possible delays, but the same ASIC is used on all boards.

In addition, a test system has been implemented on each front-end board: a LVDS signal is received on the board, translated in TTL which, and inverted, if appropriate, following the polarity associated to the board. Then, a buffer allows to send an analogue pulse to each of the 8 ASIC channels, simulating a RPC pulse with a width of 20 ns.

An adaptation board is associated to each front-end board at the other extremity of the strips. It is a simple $50 \Omega$ resistor which avoids signal reflection along the strips.

\section{RADIATION TESTS}

Tests have been performed to check the radiation hardness in conditions close (or worse) than the expected ALICE working

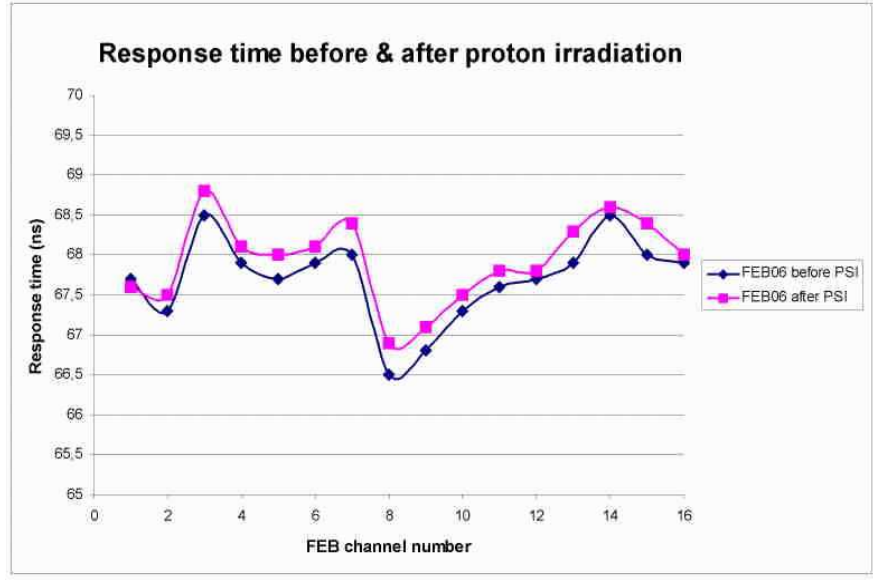

Fig. 5

RESPONSE TIME FOR 16 CHANNELS (2 ASICS) BEFORE AND AFTER IRRADIATION WITH THE PROTON BEAM AT PSI-ZÜRICH.

environment:

- Cumulative effects due to low energy neutrons and to ionizing particles (such as photons) which damage progressively the electronics components. The simulations indicates 2.6 Gy over 10 LHC years for the boards closest to the beam.

- Single event effects due to energy hadrons $(>30 \mathrm{MeV})$ which induce malfunctioning or may even destroy components. The simulations indicates $2 \times 10^{11}$ hadrons $/ \mathrm{cm}^{2}$ over 10 LHC years (and integrated over the energy spectrum) for the boards closest to the beam.

The first test was done at the Gamma Irradiation Facility (GIF) at CERN providing $\gamma$ of $662 \mathrm{keV}$ from a Cs source. The total dose received by the electronics was about $0.25 \mathrm{~Gy}$. A second test was performed at the neutron generator of the LPC Clermont-Ferrand which delivers neutrons of $14.1 \mathrm{MeV}$. The total neutron fluence received by the electronics components was $3.8 \times 10^{10} \mathrm{n} / \mathrm{cm}^{2}$ corresponding to a dose of $2.5 \mathrm{~Gy}$ which was measured with the help of PIN diodes. The third test was done with protons of $60 \mathrm{MeV}$ at the Paul Scherrer Institute (PSI) at Zürich. The fluence measured on the board was $2.3 \times 10^{11} \mathrm{p} / \mathrm{cm}^{2}$.

During all these tests, the front-end boards were active and pulsed signals from a generator (or the internal test system of the board) were sent (or activated) to check the ASIC (or the whole chain: test system plus ASIC) for each electronics channel. For the three campaigns, no problem has been observed, as illustrated for example by Fig. [5] showing the response time obtained with two ASICs of the same board before and after irradiation to protons at PSI.

\section{FRONT-END ELECTRONICS PRODUCTION}

The main constraint on this front-end electronics is to deliver the signal of any of the 21000 channels to the muon trigger electronics in a time window less than $25 \mathrm{~ns}$ (corresponding to 


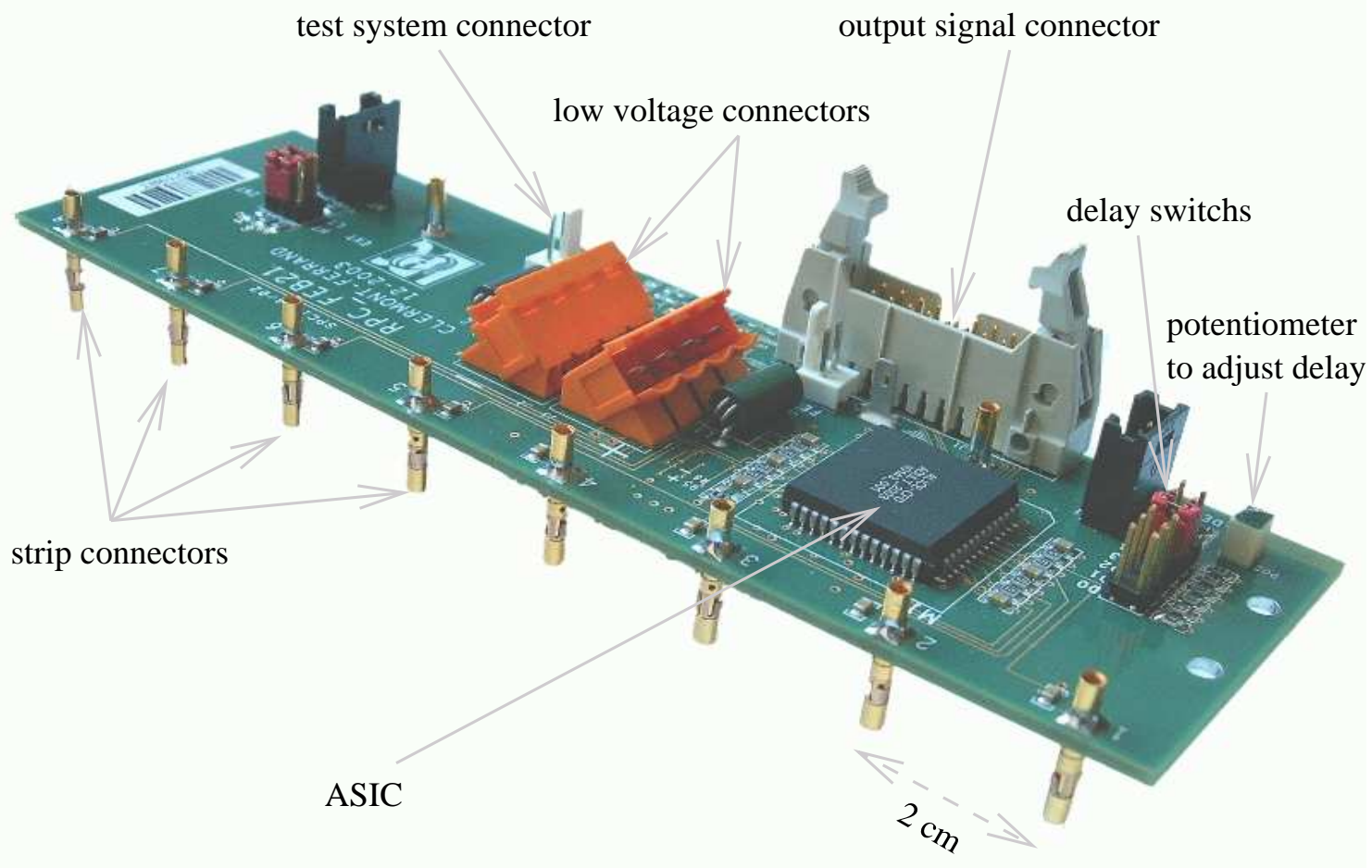

Fig. 4

PICTURE OF A FRONT-END BOARD CORRESPONDING TO 2 CM WIDE STRIPS.

the LHC clock). By taking into account all the time dispersion sources coming mainly from the RPC itself, the strip length (up to $72 \mathrm{~cm}$ ), the output signal cables (up to 20 meters), the requirement for the front-end electronics is a time dispersion less than 4 ns.

To check this time requirements and others parameters of each electronics channels, the test of the production is divided in two steps:

(i) a working test and characterization of each ASIC (whole production done end of the year 2003 corresponding to 3880 chips),

(ii) a tuning of the response time of the board (mean value) with the help of a potentiometer and a measurement of each parameters (about 12) of each electronics channel of the front-end boards (production done during summer 2004).

\section{A. Automatic test bench design}

The test bench is based on a special card equipped with relays which allow to switch to any electronics input and output channel of a board (the six different mechanical front-end boards can be handled). The input signal is generated by a pulse generator simulating RPC signals, including precursor and streamer peaks, with the possibility to vary each parameters of the signal (amplitude of each peak, time jitter between precursor and streamer, ...). A copy of this signal is sent to a scope
(500 $\mathrm{MHz}$ bandwidth) to measure exactly its characteristics. The scope is also used to measure the output LVDS signal generated by the front-end board. The apparatus of the test bench are controlled by Labview via GPIB and the relays via a DIO card.

\section{B. Test of the ASIC production}

In practice, for the test of the ASICs, the same front-end board equipped with a socket allowing to plug and unplug easily each chip was used. For each ASIC, the 8 output signals were characterized by measuring the amplitude of the LVDS signal $A_{c h}$, its width $w_{c h}$, and the time difference between the slowest and the fastest channels $\Delta t_{A S I C}$ (which can be associated to the response time dispersion of the ASIC). Fig. 6 shows the channel output signal width distribution and the ASIC time dispersion. The requirements for each parameter are: $A_{c h}=$ $(800 \pm 100) \mathrm{mV}, w_{c h}=(23.0 \pm 1.5) \mathrm{ns}$ and $\Delta t_{A S I C}<3 \mathrm{~ns}$.

Over 3880 ASICs, the results are the following:

- $5.7 \%$ were not working (due to short-circuits, ...),

- $11.8 \%$ were working but with at least one parameter outside limits (about half due to output signal width and half due to response time dispersion),

- $82.5 \%$ were within specifications.

This means that 3280 ASICs are available for ALICE front-end board production (while 2624 are needed). 


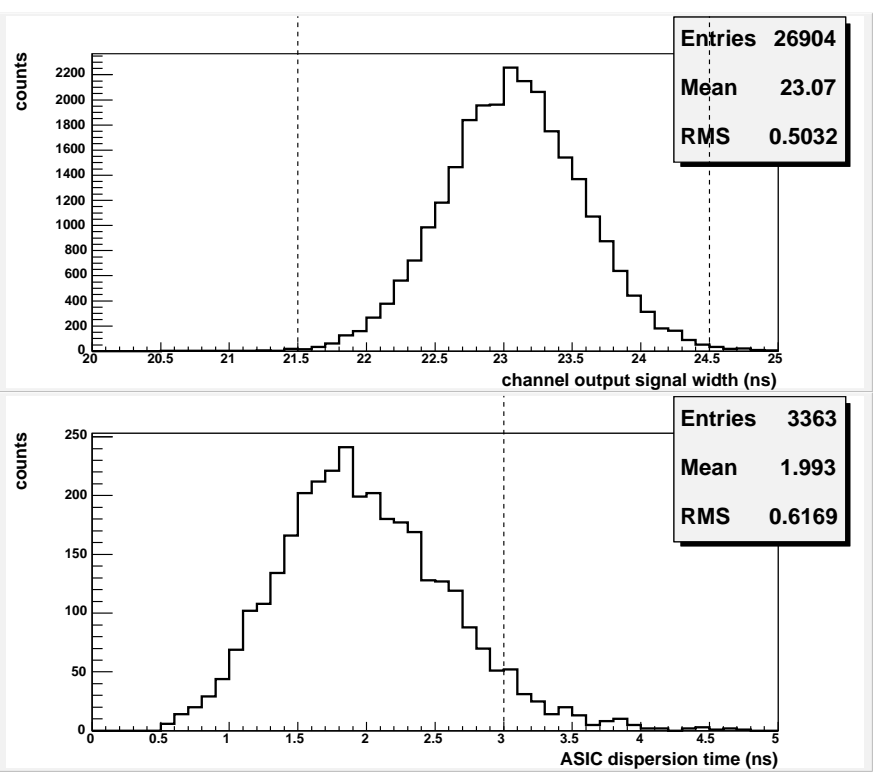

Fig. 6

ASIC PRODUCTION CHARACTERISTICS: CHANNEL OUTPUT SIGNAL WIDTH $w_{c h}$ (UPPER PLOT) AND ASIC DISPERSION TIME $\Delta t_{A S I C}$ (LOWER PLOT). THE DOTTED LINES ON EACH PLOT REPRESENT THE REQUIREMENTS.

\section{Test of the pre-production boards}

The whole production of the boards (including spares) was done during summer 2004. At the time of the writing, the tests have just started and are scheduled to finish in summer 2005.

However, the test of a pre-production representing about $12 \%$ the whole production (286 boards) was performed at the beginning of 2004. These boards are devoted to the test bench of the RPCs located at Torino. Each board has been tested and all the parameters measured. Fig. 7 displays the results obtained for three parameters: the response time to the internal test system, the low and high threshold discriminator. The most crucial parameter, e.g. the response time, shows good behavior (the RMS of the distribution is less than $1 \mathrm{~ns}$ ) with respect to the ALICE requirement (all 21000 channels in a time window of $4 \mathrm{~ns}$ due to the front-end dispersion alone). The value of the discriminator thresholds are relatively well peaked around the expectations.

During the test, it appeared that 5 ASICs were not working over 316 (among the 286 boards, 30 have two ASICs), namely about $2 \%$. The other problems met are attributed to the cabling and concerned about $4 \%$ of the boards (only one problem on printed circuit was reported). After intervention, only $2 \%$ of the board have at least one channel with one or more parameters outside specifications. In this case, the only possibility to solve the problem was to change the ASIC.

\section{CONCLUSION}

The dedicated front-end electronics of the ALICE muon trigger, for RPC in streamer mode, is based on a 8 channels

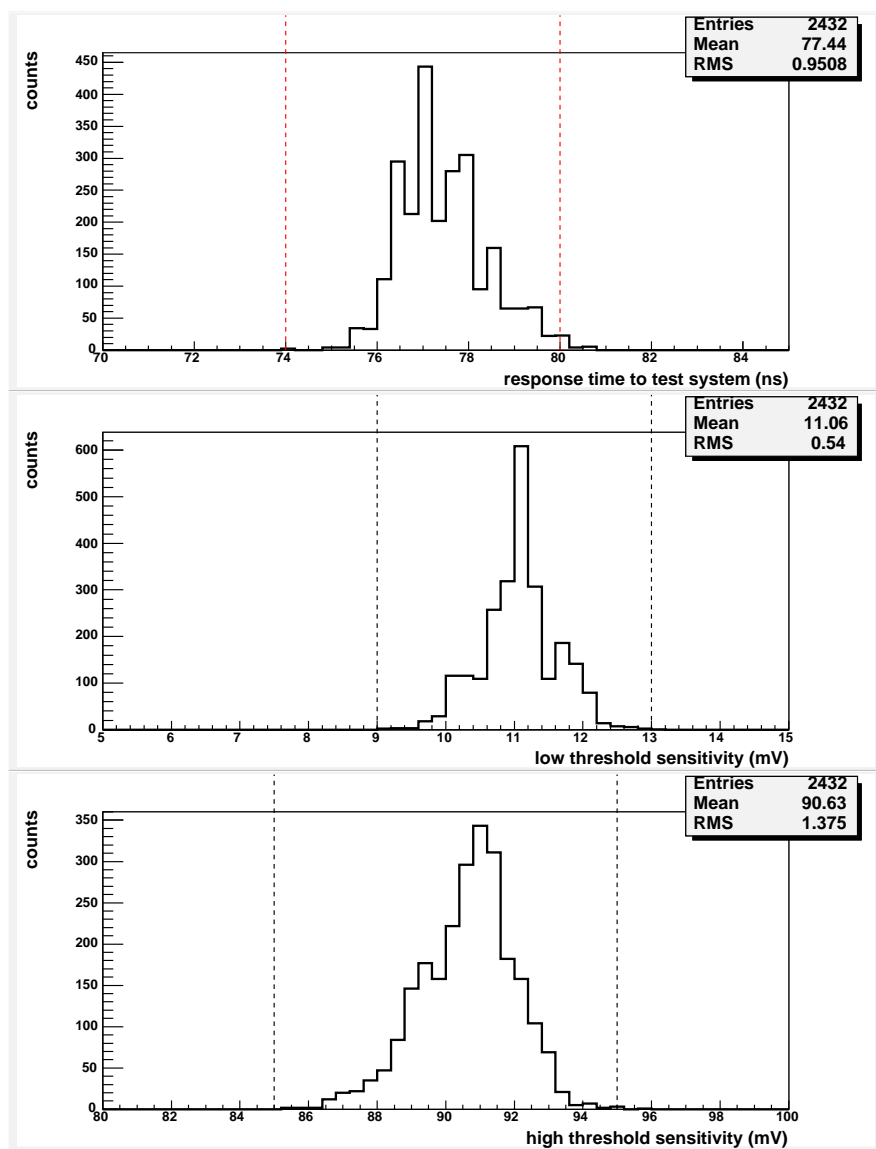

Fig. 7

FRONT-END BOARDS PRE-PRODUCTION CHARACTERISTICS: RESPONSE TIME WITH THE INTERNAL TEST SYSTEM (UPPER PLOT), LOW THRESHOLD DISCRIMINATOR (MIDDLE PLOT) AND HIGH THRESHOLD DISCRIMINATOR

(LOWER PLOT). THE DOTTED LINES ON EACH PLOT REPRESENT THE REQUIREMENTS.

ASIC which has been produced and tested. Its performance fulfils the ALICE requirements, both in terms of timing and concerning the low threshold discriminator sensitivity needed for the ADULT technique. The yield of bad chips (less than $6 \%$ ) is acceptable. The pre-production of front-end boards supporting the ASIC shows that it is possible to tune each board such that all channels are within a time window of $4 \mathrm{~ns}$. The fraction of chips to be changed after the cabling phase is about $4 \%$, leaving a sufficient number of spares for the lifetime of the ALICE experiment.

\section{ACKNOWLEDGMENT}

The authors would like to thank the Région Auvergne (France) for funding support.

\section{REFERENCES}

[1] ALICE Collaboration, ALICE Technical Proposal, CERN/LHCC 95-71 (1995).

ALICE Collaboration, ALICE Technical Proposal (Addendum), CERN/LHCC 96-32 (1996). 
[2] ALICE Collaboration, ALICE Physics Performance Report (Volume 1), CERN/LHCC 2003-049 (2003).

[3] R. Vogt, Phys. Rept. 310 (1999) 197-260.

[4] ALICE Collaboration, ALICE Technical Design Report, CERN/LHCC 9922 (1999).

[5] R. Santonico and R. Cardarelli, Nucl. Instr. and Meth. A 187 (1981) 377380.

[6] R. Arnaldi et al., Nucl. Instr. and Meth. A 456 (2000) 462-473.

[7] ALICE Collaboration, ALICE Technical Design Report, CERN/LHCC 2003-062 (2003).

[8] R. Arnaldi et al., Nucl. Instr. and Meth. A 457 (2001) 117-125.

P. Dupieux, Nucl. Instr. and Meth. A 508 (2003) 185-188.

[9] L. Royer et al., Proceeding of the 6th Workshop on electronics for the LHC experiments, CERN/LHCC 2000-041 (2000) 323-327. 


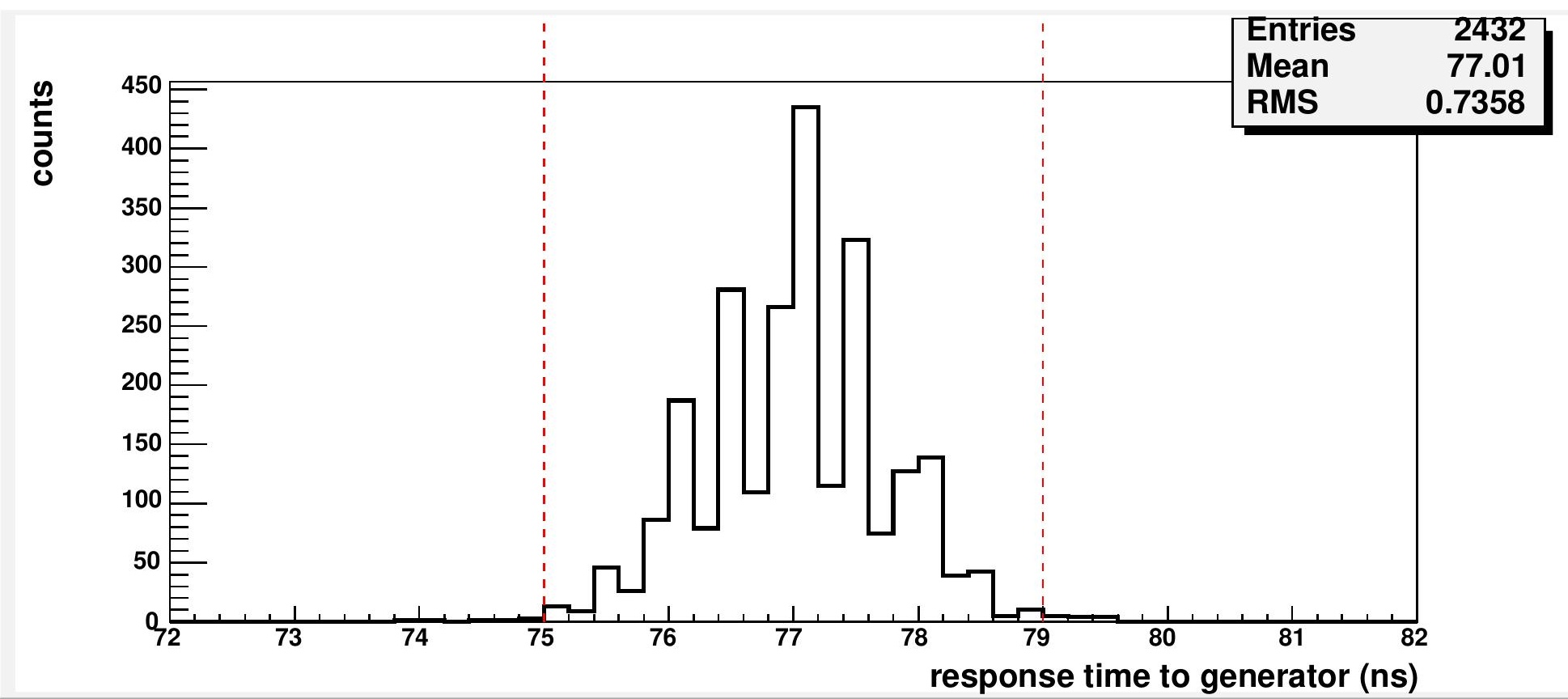

